\title{
Characterizing Classes of Regular Languages Using Prefix Codes of Bounded Synchronization Delay
}

\author{
Volker Diekert ${ }^{1}$ and Tobias Walter*†2 \\ 1 University of Stuttgart, FMI, Stuttgart, Germany \\ diekert@fmi.uni-stuttgart.de \\ 2 University of Stuttgart, FMI, Stuttgart, Germany \\ walter@fmi.uni-stuttgart.de
}

\begin{abstract}
In this paper we continue a classical work of Schützenberger on codes with bounded synchronization delay. He was interested in characterizing those regular languages where the groups in the syntactic monoid belong to a variety $\mathbf{H}$. He allowed operations on the language side which are union, intersection, concatenation and modified Kleene-star involving a mapping of a prefix code of bounded synchronization delay to a group $G \in \mathbf{H}$, but no complementation. In our notation this leads to the language classes $\operatorname{SD}_{G}\left(A^{\infty}\right)$ and $\operatorname{SD}_{\mathbf{H}}\left(A^{\infty}\right)$. Our main result shows that $\mathrm{SD}_{\mathbf{H}}\left(A^{\infty}\right)$ always corresponds to the languages having syntactic monoids where all subgroups are in $\mathbf{H}$. Schützenberger showed this for a variety $\mathbf{H}$ if $\mathbf{H}$ contains Abelian groups, only. Our method shows the general result for all $\mathbf{H}$ directly on finite and infinite words. Furthermore, we introduce the notion of local Rees extensions which refers to a simple type of classical Rees extensions. We give a decomposition of a monoid in terms of its groups and local Rees extensions. This gives a somewhat similar, but simpler decomposition than in Rhodes' synthesis theorem. Moreover, we need a singly exponential number of operations, only. Finally, our decomposition yields an answer to a question in a recent paper of Almeida and Klíma about varieties that are closed under Rees extensions.
\end{abstract}

1998 ACM Subject Classification F.4.3 Formal Languages

Keywords and phrases formal language, synchronization delay, variety, Rees extension

Digital Object Identifier 10.4230/LIPIcs.ICALP.2016.129

In memoriam: Marcel-Paul Schützenberger

(1920-1996)

\section{Introduction}

A fundamental result of Schützenberger characterizes the class of star-free languages SF as exactly those languages which are group-free, that is, aperiodic [15]. One usually abbreviates this result by $\mathrm{SF}=\mathbf{A P}$. Schützenberger also found another, but less prominent characterization of SF: the star-free languages are exactly the class of languages which can be defined inductively by finite languages and closure under finite union, concatenation, and the Kleene-star restricted to prefix codes of bounded synchronization delay [17]. This result

\footnotetext{
* Supported by the German Research Foundation (DFG) under grant DI 435/6-1.

$\dagger$ The authors thank an anonymous referee for the suggestion of the notation group-controlled star.
} 
is abbreviated by $\mathbf{A P}=\mathrm{SD}$. It is actually stronger than the famous $\mathrm{SF}=\mathbf{A P}$ because $\mathrm{SD} \subseteq \mathrm{SF} \subseteq \mathbf{A P}$ is relatively easy, see [11, Chapter $\mathrm{VIII}]$, so $\mathrm{SF}=\mathbf{A P}$ follows from $\mathbf{A P} \subseteq \mathrm{SD}$. The extension $\mathbf{S F}=\mathbf{A P}$ to infinite words is due to Perrin [10]. The result $\mathbf{A P}=\mathrm{SD}$ for infinite words was obtained much later in [5]. It became possible thanks to a "local divisor approach", which also is a main tool in this paper.

Schützenberger did not stop by showing $\mathbf{A P}=\mathrm{SD}$. In retrospective he started a program: in [16] he was able to prove an analogue of $\mathbf{A P}=\mathrm{SD}$ for languages where syntactic monoids have Abelian subgroups, only. In our notation $\mathbf{A P}=\mathrm{SD}$ means $\overline{\mathbf{1}}\left(A^{\infty}\right)=\mathrm{SD}_{\mathbf{1}}\left(A^{\infty}\right)$; and the main result in [16] is "essentially" equivalent to $\overline{\mathbf{A b}}\left(A^{*}\right)=\operatorname{SD}_{\mathbf{A b}}\left(A^{*}\right)$. (We write "essentially" because using the structure theory of Abelian groups, a sharper version than $\overline{\mathbf{A b}}\left(A^{*}\right)=\mathrm{SD}_{\mathbf{A b}}\left(A^{*}\right)$ is possible.) The proofs [16] use deep results in semigroup theory; and no such result beyond Abelian groups was known so far. Our result generalizes $\overline{\mathbf{A b}}\left(A^{\infty}\right)=$ $\mathrm{SD}_{\mathbf{A b}}\left(A^{\infty}\right)$ to every variety $\mathbf{H}$ of finite groups: we show $\overline{\mathbf{H}}\left(A^{\infty}\right)=\mathrm{SD}_{\mathbf{H}}\left(A^{\infty}\right)$. We were able to prove it with much less technical machinery compared to [16]. For example, no knowledge in Krohn-Rhodes theory is required.

Actually, our result is a generalization of $\overline{\mathbf{A b}}\left(A^{*}\right)=\mathrm{SD}_{\mathbf{A b}}\left(A^{*}\right)[16]$ and also of $\mathbf{A P}\left(A^{\infty}\right)=$ $\mathrm{SD}\left(A^{\infty}\right)$ [5]. More precisely, we give a characterization of languages which are recognized by monoids where all subgroups belong to $\mathbf{H}$. The characterization uses an inductive scheme starting with all finite subsets of finite words, allows concatenation, finite union, no (!) complementation, but a restricted use of a group-controlled star (resp. group-controlled $\omega$-power). Let us explain the group-controlled star in our context. Instead of putting the star above a single language, consider first a disjoint union $K=\bigcup\left\{K_{g} \mid g \in G\right\}$ where $G$ is a finite group and each $K_{g}$ is regular in $A^{*}$. The "group-controlled star", more precisely the "G-controlled star", associates with such a disjoint union the following language:

$$
\left\{u_{g_{1}} \cdots u_{g_{k}} \in K^{*} \mid u_{g_{i}} \in K_{g_{i}} \wedge g_{1} \cdots g_{k}=1 \in G\right\} .
$$

Clearly, we obtain a regular language, but without any restriction, allowing such a "group star" yields all regular languages, even in the case of the trivial group. So, the construction is of no interest without a simultaneous restriction. The restriction considered in [16] yields an inductive scheme to define a class $\mathcal{C}$. The restriction says that such a group-controlled star is allowed only over a disjoint union $K=\bigcup\left\{K_{g} \mid g \in G\right\}$ where each $K_{g}$ already belongs to $\mathcal{C}$ and where $K$ is, in addition, a prefix code of bounded synchronization delay. The initials in "synchronization delay" led to the notation $\mathrm{SD}$; and an indexed version $\mathrm{SD}_{G}$ (resp. $\left.\mathrm{SD}_{\mathbf{H}}\right)$ refers to synchronization delay over $G$ (resp. over a finite group in $\mathbf{H}$ ). Since we also deal with infinite words we apply the same restriction to $\omega$-powers.

Our results give also a new characterization for various other classes. For example, by a result of Straubing, Thérien and Thomas [20], the class of languages, having syntactic monoids where all subgroups are solvable, coincides with $(\mathrm{FO}+\mathrm{MOD})[<]$. Here, $(\mathrm{FO}+\mathrm{MOD})[<]$ means the class of languages defined by the logic $(\mathrm{FO}+\mathrm{MOD})[<]$. Thus, we are able to give a new language characterization: $(\mathrm{FO}+\mathrm{MOD})[<]\left(A^{\infty}\right)=\mathrm{SD}_{\mathrm{Sol}}\left(A^{\infty}\right)$.

Moreover, as a sort of byproduct of $\overline{\mathbf{H}}=\mathrm{SD}_{\mathbf{H}}$, we obtain a simple and purely algebraic characterization of the monoids in $\overline{\mathbf{H}}$. Every monoid in $\overline{\mathbf{H}}$ can be decomposed in at most exponentially many iterated Rees extensions of groups in $\mathbf{H}$. The iteration uses only a very restricted version of Rees extensions: local Rees extensions. This means we obtain every finite monoid which is not a group as a divisor of a Rees extension between two proper divisors of $M$, one of them a proper submonoid, the other one a "local divisor".

Our decomposition result is similar to the synthesis theory of Rhodes and Allen [13]. Moreover, it yields a singly exponential bound on the number of operations whereas no such 
bound was known by [13]. Finally, using this decomposition, we answer a recent question of Almeida and Klíma [1] concerning varieties which are closed under Rees extensions.

\section{Preliminaries}

Throughout, $A$ denotes a finite alphabet and $A^{*}$ is the free monoid over $A$. It consists of all finite words. The empty word is denoted by 1 as the neutral elements in other monoids or groups. The set of non-empty finite words is $A^{+}$; it is the free semigroup over $A$. By $A^{\omega}$ we denote the set of all infinite words with letters in $A$. For a set $K \subseteq A^{*}$, we let $K^{\omega}=\left\{u_{1} u_{2} \cdots \mid u_{i} \in K, u_{i}\right.$ non-empty, $\left.i \in \mathbb{N}\right\} \subseteq A^{\omega}$. In particular, $K^{\omega}=(K \backslash\{1\})^{\omega}$. Since our results concern finite and infinite words, it is convenient to treat finite and infinite words simultaneously. We define $A^{\infty}=A^{*} \cup A^{\omega}$ to be the set of finite or infinite words. Accordingly, a language $L$ is a subset of $A^{\infty}$. We say that $L$ is regular, if first, $L \cap A^{*}$ is regular and second, $L \cap A^{\omega}$ is $\omega$-regular in the standard meaning of formal language theory. In order to study regular languages algebraically, one considers finite monoids. A divisor of a monoid $M$ is a monoid $N$ which is a homomorphic image of a subsemigroup of $M$. In this case we write $N \preceq M$. A subsemigroup $S$ of $M$ is in our setting a divisor if and only if $S$ is a monoid (but not necessarily a submonoid of $M$ ). A variety of finite monoids - hence, in Birkhoff's setting: a pseudovariety - is a class of finite monoids $\mathbf{V}$ which is closed under finite direct products and under division:

- If $I$ is a finite index set and $M_{i} \in \mathbf{V}$ for each $i \in I$, then $\prod_{i \in I} M_{i} \in \mathbf{V}$. In particular, the trivial group $\{1\}$ belongs to $\mathbf{V}$.

- If $M \in \mathbf{V}$ and $N \preceq M$, then $N \in \mathbf{V}$.

Classical formal language theory states "regular" is the same as "recognizable". This means: $L \subseteq A^{*}$ is regular if and only if its syntactic monoid is finite; $L \subseteq A^{\omega}$ is regular if and only if its syntactic monoid in the sense of Arnold [2] is finite and, in addition, $L$ is saturated by the syntactic congruence, see eg. $[11,21]$. Here we use a notion of recognizability which applies to languages $L \subseteq A^{\infty}$. Let $\varphi: A^{*} \rightarrow M$ be a homomorphism to a finite monoid $M$ First, we define a relation $\sim_{\varphi}$ as follows. If $u \in A^{*}$ is a finite word, then we write $u \sim_{\varphi} v$ if $v$ is finite and $\varphi(u)=\varphi(v)$. If $u \in A^{\omega}$ is an infinite word, then we write $u \sim_{\varphi} v$ if $v$ is infinite and if there are factorizations $u=u_{1} u_{2} \cdots$ and $v=v_{1} v_{2} \cdots$ into finite nonempty words such that $\varphi\left(u_{i}\right)=\varphi\left(v_{i}\right)$ for all $i \geq 1$. It is easy to see that $\sim_{\varphi}$ is not transitive on infinite words, in general. Therefore, we consider its transitive closure $\approx_{\varphi}$. If $u, v \in A^{*}$, then we have

$$
u \sim_{\varphi} v \Longleftrightarrow u \approx_{\varphi} v \Longleftrightarrow \varphi(u)=\varphi(v)
$$

If $\alpha, \beta \in A^{\omega}$, then we have $\alpha \approx_{\varphi} \beta$ if and only if there is sequence of infinite words $\alpha_{0}, \ldots \alpha_{k}$ such that

$$
\alpha=\alpha_{0} \sim_{\varphi} \cdots \sim_{\varphi} \alpha_{k}=\beta
$$

We say that $L \subseteq A^{\infty}$ is recognizable by $M$ if there exists a homomorphism $\varphi: A^{*} \rightarrow M$ such that $u \in L$ and $u \sim_{\varphi} v$ implies $v \in L$. We also say that $M$ or $\varphi$ recognizes $L$ in this case. The connection to the classical notation is as follows. A regular language $L \subseteq A^{\infty}$ is recognizable (in our sense) by $\varphi$ if and only if the syntactic monoids of $L \cap A^{*}$ and $L \cap A^{\omega}$ are divisors of $M$. Another equivalent definition can be given in terms of Wilke algebras [22].

Every variety $\mathbf{V}$ defines a family of regular languages $\mathbf{V}\left(A^{\infty}\right)$ as follows: we let $L \in$ $\mathbf{V}\left(A^{\infty}\right)$ if there exists a monoid $M \in \mathbf{V}$ which recognizes $L$. Further, we define $\mathbf{V}\left(A^{*}\right)=$ $\left\{L \subseteq A^{*} \mid L \in \mathbf{V}\left(A^{\infty}\right)\right\}$ and $\mathbf{V}\left(A^{\omega}\right)=\left\{L \subseteq A^{\omega} \mid L \in \mathbf{V}\left(A^{\infty}\right)\right\}$. A variety of finite groups is 
a variety of finite monoids which contains only groups. Throughout $\mathbf{H}$ denotes a variety of finite groups. Special cases are the varieties

- 1: the trivial group $\{1\}$, only.

- Ab: all finite Abelian groups.

- Sol: all finite solvable groups.

- $\mathbf{S o l}_{q}$ : all finite solvable groups where the order is divisible by some power of $q$.

- G: all finite groups.

According to standard notation $\overline{\mathbf{H}}$ denotes the variety of finite monoids where all subgroups belong to $\mathbf{H}$. It is not completely obvious, but a classical fact [9], that $\overline{\mathbf{H}}$ is indeed a variety. In fact, it is the maximal variety $\mathbf{V}$ such that $\mathbf{V} \cap \mathbf{G}=\mathbf{H}$.

Clearly, $\overline{\mathbf{G}}$ is the class of all finite monoids. The most prominent subclass is $\overline{\mathbf{1}}$ : it is the variety of aperiodic monoids $\mathbf{A P}$. The class $\mathbf{A P}\left(A^{\infty}\right)=\overline{\mathbf{1}}\left(A^{\infty}\right)$ admits various other characterizations as subsets of $A^{\infty}$. For example, it is the class of star-free languages $\operatorname{SF}\left(A^{\infty}\right)$, it is the class of first-order definable languages, and it is the class of definable languages in linear temporal logic over finite or infinite words: $\operatorname{LTL}\left(A^{\infty}\right)$.

Local divisors. Let $M$ be a finite monoid and $c \in M$. Consider the set $c M \cap M c$ with a new multiplication o which is defined as follows:

$m c \circ c n=m c n$.

A straightforward calculation shows that $c M \cap M c$ becomes a monoid with this operation where the neutral element of $M_{c}$ is $c$. Thus, the structure $M_{c}=(c M \cap M c, \circ, c)$ defines a monoid. We say that $M_{c}$ is the local divisor of $M$ at $c$. If $c$ is a unit, then $M_{c}$ is isomorphic to $M$. If $c=c^{2}$, then $M_{c}$ is the standard "local monoid" at the idempotent $c$.

The important fact is that $M_{c}$ is always a divisor of $M$ and that $\left|M_{c}\right|<|M|$ as soon as $c$ is not a unit of $M$. Indeed, the mapping $\lambda_{c}:\{x \in M \mid c x \in M c\} \rightarrow M_{c}$ given by $\lambda_{c}(x)=c x$ is a surjective homomorphism. Moreover, if $c$ is not a unit, then $1 \notin c M \cap M c$, hence $\left|M_{c}\right|<|M|$. Thus, if $M$ belongs to some variety $\mathbf{V}$, then $M_{c}$ belongs to the same variety. If $M$ is not a group, then we find some nonunit $c \in M$ and the local divisor $M_{c}$ is smaller than $M$. This makes the construction useful for induction. For a survey on the local divisor technique we refer to [6].

Rees extensions. Let $N, L$ be monoids and $\rho: N \rightarrow L$ be any mapping. The Rees extension $\operatorname{Rees}(\mathrm{N}, \mathrm{L}, \rho)$ is a classical construction for monoids $[12,14]$, frequently described in terms of matrices. Here, we use an equivalent definition as in [7]. As a set we define

$$
\operatorname{Rees}(\mathrm{N}, \mathrm{L}, \rho)=N \cup(N \times L \times N) .
$$

The multiplication - on $\operatorname{Rees}(\mathrm{N}, \mathrm{L}, \rho)$ is given by

$$
\begin{aligned}
n \cdot n^{\prime} & =n n^{\prime} & & \text { for } n, n^{\prime} \in N, \\
n \cdot\left(n_{1}, m, n_{2}\right) \cdot n^{\prime} & =\left(n n_{1}, m, n_{2} n^{\prime}\right) & & \text { for } n, n^{\prime}, n_{1}, n_{2} \in N, m \in L, \\
\left(n_{1}, m, n_{2}\right) \cdot\left(n_{1}^{\prime}, m^{\prime}, n_{2}^{\prime}\right) & =\left(n_{1}, m \rho\left(n_{2} n_{1}^{\prime}\right) m^{\prime}, n_{2}^{\prime}\right) & & \text { for } n_{1}, n_{1}^{\prime}, n_{2}, n_{2}^{\prime} \in N, m, m^{\prime} \in L .
\end{aligned}
$$

The neutral element of $\operatorname{Rees}(\mathrm{N}, \mathrm{L}, \rho)$ is $1 \in N$ and $N \subseteq \operatorname{Rees}(\mathrm{N}, \mathrm{L}, \rho)$ is an embedding of monoids. In general, $L$ is not a divisor of $\operatorname{Rees}(\mathrm{N}, \mathrm{L}, \rho)$. The following property holds.

- Lemma 1. Let $N \preceq N^{\prime}$ and $L \preceq L^{\prime}$. Given $\rho: N \rightarrow L$, there exists a mapping $\rho^{\prime}: N^{\prime} \rightarrow L^{\prime}$ such that $\operatorname{Rees}(\mathrm{N}, \mathrm{L}, \rho)$ is a divisor of $\operatorname{Rees}\left(\mathrm{N}^{\prime}, \mathrm{L}^{\prime}, \rho^{\prime}\right)$. 
Proof. First, assume that $N$ (resp. $L$ ) is submonoid in $N^{\prime}$ (resp. $L^{\prime}$ ). Let $\rho^{\prime}: N^{\prime} \rightarrow L^{\prime}$ be any function such that $\left.\rho^{\prime}\right|_{N}=\rho$. The mapping $\pi: \operatorname{Rees}(\mathrm{N}, \mathrm{L}, \rho) \rightarrow \operatorname{Rees}\left(\mathrm{N}^{\prime}, \mathrm{L}^{\prime}, \rho^{\prime}\right)$ given by $\pi(n)=n$ and $\pi\left(n_{1}, \ell, n_{2}\right)=\left(n_{1}, \ell, n_{2}\right)$ is an injective homomorphism.

Second, let $\varphi: N^{\prime} \rightarrow N$ and $\psi: L^{\prime} \rightarrow L$ be surjective homomorphisms. Let $\rho^{\prime}: N^{\prime} \rightarrow L^{\prime}$ be a function such that $\rho^{\prime}(n) \in \psi^{-1}(\rho(\varphi(n)))$. Let $\pi: \operatorname{Rees}\left(\mathrm{N}^{\prime}, \mathrm{L}^{\prime}, \rho^{\prime}\right) \rightarrow \operatorname{Rees}(\mathrm{N}, \mathrm{L}, \rho)$ be the mapping defined by $\pi(n)=\varphi(n)$ and $\pi\left(n_{1}, \ell, n_{2}\right)=\left(\varphi\left(n_{1}\right), \psi(\ell), \varphi\left(n_{2}\right)\right)$. It is clear that $\pi$ is surjective. It is a homomorphism since

$$
\begin{gathered}
\pi\left(\left(n_{1}, \ell, n_{2}\right) \cdot\left(n_{1}^{\prime}, \ell^{\prime}, n_{2}^{\prime}\right)\right)=\pi\left(n_{1}, \ell \rho^{\prime}\left(n_{2} n_{1}^{\prime}\right) \ell^{\prime}, n_{2}^{\prime}\right)=(\varphi\left(n_{1}\right), \psi(\ell) \underbrace{\psi\left(\rho^{\prime}\left(n_{2} n_{1}^{\prime}\right)\right)}_{=\rho\left(\varphi\left(n_{2} n_{1}^{\prime}\right)\right)} \psi\left(\ell^{\prime}\right), \varphi\left(n_{2}^{\prime}\right)) \\
=\left(\varphi\left(n_{1}\right), \psi(\ell), \varphi\left(n_{2}\right)\right) \cdot\left(\varphi\left(n_{1}^{\prime}\right), \psi\left(\ell^{\prime}\right), \varphi\left(n_{2}^{\prime}\right)\right)=\pi\left(n_{1}, \ell, n_{2}\right) \cdot \pi\left(n_{1}^{\prime}, \ell^{\prime}, n_{2}^{\prime}\right)
\end{gathered}
$$

We are mainly interested in the case where $N$ and $L$ are proper divisors of a given finite monoid $M$. This leads to the notion of local Rees monoids. More precisely, let $M$ be a finite monoid, $N$ be a proper submonoid of $M$ and $M_{c}$ be a local divisor of $M$ at $c$ where $c$ is not a unit. The local Rees extension $\operatorname{LocRees}\left(\mathrm{N}, \mathrm{M}_{\mathrm{c}}\right)$ is defined as the Rees extension $\operatorname{Rees}\left(\mathrm{N}, \mathrm{M}_{\mathrm{c}}, \rho_{\mathrm{c}}\right)$ where $\rho_{c}$ denotes the mapping $\rho_{c}: N \rightarrow M_{c} ; x \mapsto c x c$.

For a variety $\mathbf{V}$ we define $\operatorname{Rees}(\mathbf{V})$ to be the least variety which contains $\mathbf{V}$ and is closed under taking Rees extensions and $\operatorname{LocRees}(\mathbf{V})$ to be the least variety which contains $\mathbf{V}$ and is closed under local Rees extensions.

\subsection{Schützenberger's SD classes}

Schützenberger gave a language theoretical characterization of the class of star-free languages $\mathrm{SF}\left(A^{*}\right)$ avoiding complementation, but allowing the star-operation to prefix codes of bounded synchronization delay [17].

A language $K \subseteq A^{+}$is called prefix code if it is prefix-free. That is: $u, u v \in K$ implies $u=u v$. A prefix-free language $K$ is a code since every word $u \in K^{*}$ admits a unique factorization $u=u_{1} \cdots u_{k}$ with $k \geq 0$ and $u_{i} \in K$. Note that the empty set $\emptyset$ is considered to be a prefix code. More generally, if $L \subseteq A^{+}$is any subset, then $K=L \backslash L A^{+}$is a prefix code. A prefix code $K$ has bounded synchronization delay if for some $d \in \mathbb{N}$ and for all $u, v, w \in A^{*}$ we have: if $u v w \in K^{*}$ and $v \in K^{d}$, then $u v \in K^{*}$. Note that the condition implies that for all $u v w \in K^{*}$ with $v \in K^{d}$, we have $w \in K^{*}$, too. If $d$ is given explicitly, $K$ is said to have synchronization delay $d$. Every subset $B \subseteq A$ (including the empty set) yields a prefix code with synchronization delay 0 . If we have $c \in A \backslash B$, then $B^{*} c$ is a prefix code with synchronization delay 1 . If $K$ is any prefix code with (or without) bounded synchronization delay, then $K^{m}$ is a prefix code for all $m \in \mathbb{N}$, but for $m \geq 2$ it is never of bounded synchronization delay.

Consider a disjoint union $K=\bigcup\left\{K_{g} \mid g \in G\right\}$ of a prefix code $K$ with bounded synchronization delay where $G$ is a finite group and each $K_{g}$ is regular in $A^{*}$. The $G$-controlled star associates with such a disjoint union the following language:

$$
\left\{u_{g_{1}} \cdots u_{g_{k}} \in K^{*} \mid u_{g_{i}} \in K_{g_{i}} \wedge g_{1} \cdots g_{k}=1 \in G\right\} .
$$

Another view of the $G$-controlled star of $K$ is the following: Let $\gamma_{K}: K \rightarrow G$ be a mapping such that $K_{g}=\gamma_{K}^{-1}(g)$ and let $\gamma: K^{*} \rightarrow G$ denote the canonical extension of $\gamma_{K}$ to a homomorphism from the free submonoid $K^{*} \subseteq A^{*}$ to $G$, then the $G$-controlled star of $K$ is exactly the set $\gamma^{-1}(1)$. The generalization to infinite words $\gamma^{-1}(1)^{\omega}$ is called the $G$-controlled $\omega$-power. Let $\mathcal{C}$ be a class of languages. We say that $\mathcal{C}$ is closed under $G$-controlled star ( $\omega$-power) if $K$ is a prefix code with bounded synchronization delay, $K_{g} \in \mathcal{C}$ for all $g \in G$, 
then the $G$-controlled star $\gamma^{-1}(1)\left(\omega\right.$-power $\left.\gamma^{-1}(1)^{\omega}\right)$ is in $\mathcal{C}$. For a variety of groups $\mathbf{H}$ we say that $\mathcal{C}$ is closed under $\mathbf{H}$-controlled star ( $\omega$-power) if $\mathcal{C}$ is closed under $G$-controlled star ( $\omega$-power) for every group $G \in \mathbf{H}$. By $\mathrm{SD}_{G}\left(A^{\infty}\right)$ we denote the smallest class of regular languages such that $\emptyset \in \mathrm{SD}_{G}\left(A^{\infty}\right),\{a\} \in \mathrm{SD}_{G}\left(A^{\infty}\right)$ for all letters $a \in A, \operatorname{SD}_{G}\left(A^{\infty}\right)$ is closed under finite union and concatenation, i.e., $L, K \in \mathrm{SD}_{G}\left(A^{\infty}\right)$ implies $L \cup K$ and $\left(L \cap A^{*}\right) \cdot K$ are both in $\mathrm{SD}_{G}\left(A^{\infty}\right)$, and $\mathrm{SD}_{G}\left(A^{\infty}\right)$ is closed under $G$-controlled star and $G$-controlled $\omega$-power. We also define

$$
\operatorname{SD}_{G}\left(A^{*}\right)=\left\{L \subseteq A^{*} \mid L \in \operatorname{SD}_{G}\left(A^{\infty}\right)\right\} \quad \text { and } \quad \operatorname{SD}_{G}\left(A^{\omega}\right)=\left\{L \subseteq A^{\omega} \mid L \in \operatorname{SD}_{G}\left(A^{\infty}\right)\right\} .
$$

Note that for every homomorphism $\gamma: A^{*} \rightarrow G$ we have $\gamma^{-1}(1) \in \operatorname{SD}_{G}\left(A^{*}\right)$ and $\gamma^{-1}(1)^{\omega} \in$ $\operatorname{SD}_{G}\left(A^{\omega}\right)$. This follows because first, $A$ is a prefix code of bounded synchronization delay and second, all finite subsets of $A$ are in $\operatorname{SD}_{G}\left(A^{*}\right)$.

Unlike the case of star-free sets, the definition of $\operatorname{SD}_{G}\left(A^{\infty}\right)$ does not use any complementation. By induction: for $L \subseteq A^{\infty}$ we have $L \in \operatorname{SD}_{G}\left(A^{\infty}\right)$ if and only if we can write $L=L_{1} \cup L_{2}$ with $L_{1} \in \mathrm{SD}_{G}\left(A^{*}\right)$ and $L_{2} \in \mathrm{SD}_{G}\left(A^{\omega}\right)$. In the special case where $G=\{1\}$ is the trivial group, we also simply write $\mathrm{SD}$ instead of $\mathrm{SD}_{\{1\}}$. In this case closure under $\{1\}$-controlled stars ( $\omega$-powers) can be rephrased in simpler terms as follows: If $K \in \operatorname{SD}\left(A^{*}\right)$ is a prefix code of bounded synchronization delay, then $K^{*} \in \mathrm{SD}\left(A^{*}\right)$ and $K^{\omega} \in \mathrm{SD}\left(A^{\omega}\right)$.

In [16] Schützenberger showed (using a different notation) $\operatorname{SD}_{\mathbf{H}}\left(A^{*}\right) \subseteq \overline{\mathbf{H}}\left(A^{*}\right)$, but the converse only for $\mathbf{H} \subseteq \mathbf{A b}$, see Proposition 6 for the first inclusion. Our aim is to show $\overline{\mathbf{H}}\left(A^{\infty}\right) \subseteq \mathrm{SD}_{\mathbf{H}}\left(A^{\infty}\right)$ for all $\mathbf{H}$, cf. Theorem 4 . We begin with a technical lemma.

- Lemma 2. Let $K \subseteq A^{+}$be a prefix code of bounded synchronization delay and let $\gamma: K^{*} \rightarrow G$ be a homomorphism such that $\gamma^{-1}(g) \cap K \in \operatorname{SD}_{G}\left(A^{*}\right)$ for all $g \in G$, then we have $\gamma^{-1}(g) \in \operatorname{SD}_{G}\left(A^{*}\right)$ for all $g \in G$.

Proof. For a word $w=u_{1} \cdots u_{k} \in K^{*}$ we define $P(w)=\left\{\gamma\left(u_{1} \cdots u_{i}\right) \mid 1 \leq i \leq k\right\} \subseteq G$ to be the set of prefixes of $w$ in $G$. By an induction on $|P(w)|$ we construct languages $L(w) \in \mathrm{SD}_{G}\left(A^{*}\right)$ such that $w \in L(w) \subseteq \gamma^{-1}(\gamma(w))$ and the number $\left|\left\{L(w) \mid w \in K^{*}\right\}\right|$ of such languages is finite. The base case $|P(w)|=0$ implies $g=1$. We may choose $L(w)=\gamma^{-1}(1)$ and we are done, since $\gamma^{-1}(1) \in \operatorname{SD}_{G}\left(A^{*}\right)$ by definition. Hence, we may assume $|P(w)| \geq 1$. Let $g_{1}=\gamma\left(u_{1}\right)$ and choose $i$ maximal such that $g_{1}=\gamma\left(u_{1} \cdots u_{i}\right)$. Then we have $u_{1} \cdots u_{i} \in\left(K \cap \gamma^{-1}\left(g_{1}\right)\right) \cdot \gamma^{-1}(1)$. Note that $P\left(w^{\prime}\right)=g_{1}^{-1} \cdot\left\{\gamma\left(u_{1} \cdots u_{j}\right) \mid i<j \leq k\right\}$ for $w^{\prime}=u_{i+1} \cdots u_{k}$. By choice of $i$ we have $g_{1} \notin\left\{\gamma\left(u_{1} \cdots u_{j}\right) \mid i<j \leq k\right\}$ and therefore $\left|P\left(w^{\prime}\right)\right|=\left|\left\{\gamma\left(u_{1} \cdots u_{j}\right) \mid i<j \leq k\right\}\right|<|P(w)|$. By induction there exists $L\left(w^{\prime}\right)$ and we let $L(w)=\left(K \cap \gamma^{-1}\left(g_{1}\right)\right) \cdot \gamma^{-1}(1) \cdot L\left(w^{\prime}\right)$. The number of $\left|\left\{L(w) \mid w \in K^{*}\right\}\right|$ is therefore bounded by $\sum_{i=0}^{|G|}|G|^{i}$ which is less than $|G|^{|G|+1}$. The result follows because we can write $\gamma^{-1}(g)=\bigcup\left\{L(w) \mid w \in \gamma^{-1}(g)\right\}$ and this is a finite union.

Clearly, we have for all $G$ : if $K \in \operatorname{SD}_{G}\left(A^{*}\right)$ is a prefix code of bounded synchronization delay, then $K^{*}$ and $K^{\omega}$ are both in $\operatorname{SD}_{G}\left(A^{\infty}\right)$. As a special case, using the prefix code $K=\emptyset$, it holds $K^{*}=\{1\} \in \mathrm{SD}_{G}\left(A^{\infty}\right)$. More generally, every finite language is in $\mathrm{SD}_{G}\left(A^{\infty}\right)$. Note also that for $G^{\prime} \leq G$ we have $\operatorname{SD}_{G^{\prime}}\left(A^{\infty}\right) \subseteq \operatorname{SD}_{G}\left(A^{\infty}\right)$. In particular, $\bigcup\left\{\mathrm{SD}_{G_{i}}\left(A^{\infty}\right) \mid i \in I\right\} \subseteq \mathrm{SD}_{\prod_{i \in I} G_{i}}\left(A^{\infty}\right)$ for every finite index set $I$. This inclusion holds for every divisor of $G$ as observed by the next lemma which can be proved by induction.

- Lemma 3. $\mathrm{SD}_{H}\left(A^{\infty}\right) \subseteq \mathrm{SD}_{G}\left(A^{\infty}\right)$ holds for $H \preceq G$.

We will formulate our some of results on the language classes $\mathrm{SD}_{G}\left(A^{\infty}\right)$ to obtain finer results. However, our main result concerns the language class

$$
\mathrm{SD}_{\mathbf{H}}\left(A^{\infty}\right)=\bigcup\left\{\mathrm{SD}_{G}\left(A^{\infty}\right) \mid G \in \mathbf{H}\right\} .
$$


- Theorem 4. Let $\mathbf{H}$ be a variety of finite groups. Then $\overline{\mathbf{H}}\left(A^{\infty}\right)$ is the smallest class of languages $\mathcal{C}$ closed under finite union, concatenation, $\mathbf{H}$-controlled star and $\mathbf{H}$-controlled $\omega$-power such that $\mathcal{C}$ contains all finite languages over $A^{*}$. In other words, it holds $\overline{\mathbf{H}}\left(A^{\infty}\right)=$ $\mathrm{SD}_{\mathbf{H}}\left(A^{\infty}\right)$.

- Corollary 5. $\operatorname{SD}_{\mathbf{H}}\left(A^{\infty}\right)$ is closed under complementation and intersection for every variety $\mathbf{H}$ of finite groups.

An algebraic characterization of $\overline{\mathbf{H}}$ in terms of Rees extensions will be given in Theorem 15 . The proof of Theorem 4 covers the next two sections.

\section{Closure properties of $\mathrm{SD}_{\mathrm{H}}$}

In this section we prove $\operatorname{SD}_{\mathbf{H}}\left(A^{\infty}\right) \subseteq \overline{\mathbf{H}}\left(A^{\infty}\right)$. Therefore one has to study the closure properties under the operations given in the definition of $\operatorname{SD}_{\mathbf{H}}\left(A^{\infty}\right)$, that is, one has to show that those operations do not introduce new groups.

The next proposition shows that the $\mathbf{H}$-controlled star does not introduce new groups.

- Proposition 6 ([16]). Let $K=\bigcup\left\{K_{g} \mid g \in G\right\} \subseteq A^{+}$be a prefix code of bounded synchronization delay where each $K_{g}$ is regular. Then all subgroups in the syntactic monoid of the $G$-controlled star are divisors either of $G$ or of the direct product $\prod_{g \in G} \operatorname{Synt}\left(K_{g}\right)$.

We will prove the same for $\gamma^{-1}(1)^{\omega}$, relying on Proposition 6 as a blackbox. The concept used for transfering the properties to infinite words are Birget-Rhodes expansions [3, 4]. The BirgetRhodes expansion of a monoid $M$ is the monoid $\operatorname{Exp}(\mathrm{M})=\{(X, m) \mid 1, m \in X \subseteq M\}$. The multiplication on $\operatorname{Exp}(\mathrm{M})$ is given as a semi-direct product: $(X, m) \cdot(Y, n)=(X \cup m \cdot Y, m \cdot n)$. Note that $M$ is isomorphic to the submonoid $\{(M, m) \mid m \in M\}$ of $\operatorname{Exp}(\mathrm{M})$, that is, $M$ is a divisor of $\operatorname{Exp}(\mathrm{M})$. Moreover, the following lemma shows that the Birget-Rhodes expansion has the same groups as $M$.

- Lemma 7. Every subgroup of $\operatorname{Exp}(\mathrm{M})$ is isomorphic to some group in $M$.

Proof. Let $G \subseteq \operatorname{Exp}(\mathrm{M})$ be a group contained in $\operatorname{Exp}(\mathrm{M})$ and let $(X, e) \in G$ be the unit in $G$. For every element $(Y, m) \in G$ we have $(X, e)(Y, m)=(X \cup e Y, e m)=(Y, m)$ and hence, $X \subseteq Y$. Furthermore, $(Y, m)^{|G|}=\left(Y \cup \cdots, m^{|G|}\right)=(X, e)$ and we conclude $X=Y$. Thus, $(X, m) \mapsto m$ is an injective embedding of $G$ into $M$.

The idea behind the Birget-Rhodes expansion is that it stores the seen prefixes in a set.

- Lemma 8. Let $\varphi: A^{*} \rightarrow M$ be a homomorphism and $\psi: A^{*} \rightarrow \operatorname{Exp}(\mathrm{M})$ be the homomorphism given by $\psi(a)=(\{1, \varphi(a)\}, \varphi(a))$. Let $u \in A^{*}$ and $\psi(u)=(X, \varphi(u))$. For every $m \in X$ there exists a prefix $v$ of $u$ such that $\varphi(v)=m$.

Proof. We will prove this inductively. The statement is true if $u$ is the empty word. Thus, consider $u=v a$ for some letter $a \in A$. Let $\psi(v)=(Y, \varphi(v))$, then

$$
\psi(u)=\psi(v) \cdot(\{1, \varphi(a)\}, \varphi(a))=(Y \cup\{\varphi(v), \varphi(v) \varphi(a)\}, \varphi(u)) .
$$

Inductively, we obtain prefixes of $v$, and therefore also prefixes of $u$, for all elements of $Y$. The only (potentially) new element in $X$ is $\varphi(u)$. This proves the claim.

A special kind of $\omega$-regular languages are arrow languages. Let $L \subseteq A^{*}$ be a language. We define $\vec{L}=\left\{\alpha \in A^{\omega} \mid\right.$ infinitely many prefixes of $\alpha$ are in $\left.L\right\}$ to be the arrow language 
of $L$. The set of arrow languages is exactly the set of deterministic languages [21]. The Birget-Rhodes expansion can be used to obtain a recognizing monoid for $\vec{L}$, given a monoid for $L$. For a related result see [10].

- Proposition 9. Let $L \subseteq A^{*}$ be some regular language and $\varphi: A^{*} \rightarrow M$ be a homomorphism which recognizes $L$, then $\vec{L}$ is recognized by $\operatorname{Exp}(\mathrm{M})$.

Proof. Let $\psi: A^{*} \rightarrow \operatorname{Exp}(\mathrm{M})$ be the homomorphism given by $\psi(a)=(\{1, \varphi(a)\}, \varphi(a))$. Let $\alpha \in \vec{L}$ and $\alpha \sim_{\psi} \beta$. We show that $\beta \in \vec{L}$. Let $\alpha=u_{1} u_{2} \cdots$ and $\beta=v_{1} v_{2} \cdots$ be factorizations such that $\psi\left(u_{i}\right)=\psi\left(v_{i}\right)$. Since $\alpha \in \vec{L}$, we may assume that for every $i$ there exists a decomposition $u_{i}=u_{i}^{\prime} u_{i}^{\prime \prime}$ such that $u_{1} \cdots u_{i-1} u_{i}^{\prime} \in L$. By $\psi\left(u_{i}\right)=\psi\left(v_{i}\right)$ and Lemma 8, there exists a decomposition $v_{i}=v_{i}^{\prime} v_{i}^{\prime \prime}$ such that $\varphi\left(u_{i}^{\prime}\right)=\varphi\left(v_{i}^{\prime}\right)$. Thus, $u_{1} \cdots u_{i-1} u_{i}^{\prime} \sim_{\varphi} v_{1} \cdots v_{i-1} v_{i}^{\prime}$ and therefore $v_{1} \cdots v_{i-1} v_{i}^{\prime} \in L$. This implies $\beta \in \vec{L}$.

- Proposition 10. If $L \in \operatorname{SD}_{G}\left(A^{\infty}\right)$, then all subgroups in $\operatorname{Synt}(L)$ are a divisor of a direct product of copies of $G$.

Proof. We will prove this inductively on the definition of $\operatorname{SD}_{G}\left(A^{\infty}\right)$. The cases $\emptyset \in \mathrm{SD}_{G}\left(A^{\infty}\right)$ and $\{a\} \in \mathrm{SD}_{G}\left(A^{\infty}\right)$ for all letters $a \in A$ are straightforward, as they are recognized by aperiodic monoids. Let $L, K$ be languages, such that their syntactic monoids contain only groups which are divisors of a direct product of $G$. The language $L \cup K$ is recognized by the direct product of their syntactic monoids which implies the statement. $\left(L \cap A^{*}\right) \cdot K$ is recognized by the Schützenberger product of their syntactic homomorphisms [10] and [8, Proposition 11.7.10]. The Schützenberger product does not introduce new groups [15].

Let $K \subseteq A^{+}$be a prefix code of bounded synchronization delay and $\gamma: K^{*} \rightarrow G$ be a homomorphism of the free monoid $K^{*}$ to the group $G$ such that for all $g \in G$ every subgroup of $\operatorname{Synt}\left(K \cap \gamma^{-1}(g)\right)$ is a divisor of a direct product of copies of $G$. Proposition 6 implies that every subgroup of $\operatorname{Synt}\left(\gamma^{-1}(1)\right)$ is a divisor of a direct product of copies of $G$. Note that $\gamma^{-1}(1)^{\omega}=\overrightarrow{\gamma^{-1}(1)}$ and therefore Proposition 9 and Lemma 7 imply that every subgroup of $\operatorname{Synt}\left(\gamma^{-1}(1)^{\omega}\right)$ is a divisor of a direct product of copies of $G$.

\section{The inclusion $\overline{\mathrm{H}}\left(A^{\infty}\right) \subseteq \operatorname{SD}_{\mathrm{H}}\left(A^{\infty}\right)$}

We prove that if every subgroup of $M$ is a divisor of $G$, then every language recognized by $M$ is contained in $\operatorname{SD}_{G}\left(A^{\infty}\right)$. This result is again finer than just the inequality $\overline{\mathbf{H}}\left(A^{\infty}\right) \subseteq \operatorname{SD}_{\mathbf{H}}\left(A^{\infty}\right)$. The proof works by induction on $|M|$ and on the alphabet and decomposes every $\approx_{\varphi}$-class into several sets in $\mathrm{SD}_{G}\left(A^{\infty}\right)$. As a byproduct we obtain a normal form for the languages in $\mathrm{SD}_{G}\left(A^{\infty}\right)$.

- Proposition 11. Let $L \subseteq A^{\infty}$ be recognized by $\varphi: A^{*} \rightarrow M$ and let $G$ be a group such that every subgroup of $M$ is a divisor of $G$, then $L \in \mathrm{SD}_{G}\left(A^{\infty}\right)$. Moreover, $L$ can be written as finite union

$$
L=L_{0} \cup \bigcup_{i=1}^{m} L_{i} \cdot \gamma_{i}^{-1}(1)^{\omega}
$$

for $L_{i} \in \mathrm{SD}_{G}\left(A^{*}\right)$ and $\gamma_{i}: K_{i}^{*} \rightarrow G$ for prefix codes $K_{i} \in \mathrm{SD}_{G}\left(A^{*}\right)$ of bounded synchronization delay with $\gamma_{i}^{-1}(g) \cap K_{i} \in \mathrm{SD}_{G}\left(A^{*}\right)$ for all $g \in G$. All products in the expressions of $L_{i}$ are unambiguous. 
Proof. Let $\llbracket w \rrbracket_{\varphi}=\left\{v \in A^{\infty} \mid w \approx_{\varphi} v\right\}$ be the equivalence class of $w$. Since $L$ is recognized by $\varphi$, it holds $L=\cup_{w \in L} \llbracket w \rrbracket_{\varphi}$. Our goal is to construct languages $L(w) \in \operatorname{SD}_{G}\left(A^{\infty}\right)$ such that

- $w \in L(w) \subseteq \llbracket w \rrbracket_{\varphi}$.

- the number of such languages is finite.

- every word in $L(w)$ starts with the same letter.

In particular, we want to saturate $\llbracket w \rrbracket_{\varphi}$ by sets in $\operatorname{SD}_{G}\left(A^{\infty}\right)$. The construction of the set $L(w)$ is by induction on $(|M|,|A|)$ with lexicographic order.

If $w=1$, then we set $L(w)=\{1\}$. This concludes the induction base $|A|=0$. Let us consider the case that $\varphi\left(A^{*}\right)$ is a group, that is, a divisor of $G$. Let $K=A$. The set $K$ is a prefix code of synchronization delay 0 and we may choose the homomorphism $\gamma=\varphi$. Note that every subset of $A$ is in $\operatorname{SD}_{G}\left(A^{*}\right)$. In particular, $K_{g}=K \cap \gamma^{-1}(g) \in \mathrm{SD}_{G}\left(A^{*}\right)$ for all $g \in \varphi\left(A^{*}\right)$. This shows $\gamma^{-1}(g)=\varphi^{-1}(g) \in \mathrm{SD}_{G}\left(A^{*}\right)$ for all $g \in \varphi\left(A^{*}\right)$ by Lemma 2 and Lemma 3. In order to satisfy the third condition let $w=a v \in a A^{*}$ for some $a \in A$ and set $L(w)=a \varphi^{-1}(\varphi(v))$. It is clear that $w \in L(w) \subseteq \llbracket w \rrbracket_{\varphi}$ and $L(w) \in \mathrm{SD}_{G}\left(A^{*}\right)$ by the above. If $w \in a A^{\omega}$, then we obtain $w \in a \varphi^{-1}(g) \varphi^{-1}(1)^{\omega}$ for some $g \in \varphi\left(A^{*}\right)$ by the pigeonhole principle. Thus, we may set $L(w)=a \varphi^{-1}(g) \varphi^{-1}(1)^{\omega}$. Note that by the definition of $\sim_{\varphi}$, the inclusion $L(w) \subseteq \llbracket w \rrbracket_{\varphi}$ holds. In particular, these cases include the induction base $|M|=1$.

In the following we assume that $\varphi\left(A^{*}\right)$ is not a group and therefore there exists a letter $c \in A$ such that $\varphi(c)$ is not a unit. Fix this letter $c \in A$ and set $B=A \backslash\{c\}$. If $w \in B^{\infty}$, the set $L(w)$ exists by induction. Let $w=u v$ with $u \in B^{*}$ and $v \in c A^{\infty}$. By induction we obtain $L(u) \in \mathrm{SD}_{G}\left(B^{\infty}\right) \subseteq \mathrm{SD}_{G}\left(A^{\infty}\right)$ and it remains to show $L(v) \in \mathrm{SD}_{G}\left(A^{\infty}\right)$. Note that the product $L(w)=L(u) \cdot L(v)$ is unambiguous. From now on we may assume $w \in c A^{\infty}$. Let us first consider the case $w=u v$ with $u \in c\left(B^{*} c\right)^{*}$ and $v \in B^{\infty}$, i.e., there are only finitely many occurrences of the letter $c$ in $w$. By induction, there exists $L(v) \in \operatorname{SD}_{G}\left(B^{\infty}\right) \subseteq \operatorname{SD}_{G}\left(A^{\infty}\right)$ and by setting $L(w)=L(u) \cdot L(v)$ it remains to construct $L(u)$.

Consider the alphabet $T=\varphi\left(B^{*}\right)=\left\{\varphi(u) \mid u \in B^{*}\right\}$. Let $M_{c}$ be the local divisor of $M$ at $\varphi(c)$. Since $M_{c}$ is a divisor of $M$, every subgroup of $M_{c}$ is a divisor of $G$. Consider the homomorphism $\psi: T^{*} \rightarrow M_{c}$ given by $\psi(\varphi(u))=\varphi($ cuc $)$ and the substitution $\sigma:\left(B^{*} c\right)^{\infty} \rightarrow$ $T^{\infty}$ with $\sigma\left(u_{1} c u_{2} c \ldots\right)=\varphi\left(u_{1}\right) \varphi\left(u_{2}\right) \cdots$. Note that

$$
\begin{aligned}
\psi\left(\sigma\left(u_{1} c u_{2} c \ldots u_{n} c\right)\right) & =\psi\left(\varphi\left(u_{1}\right) \varphi\left(u_{2}\right) \cdots \varphi\left(u_{n}\right)\right)=\varphi\left(c u_{1} c\right) \circ \varphi\left(c u_{2} c\right) \circ \cdots \circ \varphi\left(c u_{n} c\right) \\
& =\varphi\left(c u_{1} c u_{2} c \ldots c u_{n} c\right)
\end{aligned}
$$

and thus $\varphi^{-1}(m) \cap c\left(B^{*} c\right)^{*}=c \sigma^{-1}\left(\psi^{-1}(m)\right)$. Since $\left|M_{c}\right|<|M|$, we can apply induction on the monoid size and there exists a language $L\left(\sigma\left(u^{\prime}\right)\right) \in \mathrm{SD}_{G}\left(T^{\infty}\right)$ for all $u^{\prime} \in\left(B^{*} c\right)^{*}$. We set $L(u)=c \sigma^{-1}\left(L\left(\sigma\left(u^{\prime}\right)\right)\right)$ for $u=c u^{\prime}$. In order to complete the case of finitely many $c^{\prime}$ 's, it suffices to show the following claim:

Claim. It is $\sigma^{-1}(K) \in \mathrm{SD}_{G}\left(A^{\infty}\right)$ for all $K \in \mathrm{SD}_{G}\left(T^{\infty}\right)$.

Proof of the Claim: We prove the claim inductively on the definition of $\mathrm{SD}_{G}$. For $K=\emptyset$, we obtain $\sigma^{-1}(K)=\emptyset \in \operatorname{SD}_{G}\left(A^{\infty}\right)$. Furthermore,

$$
\sigma^{-1}(t)=\bigcup_{v \in B^{*}, t=\varphi(v)} L(v) c \in \operatorname{SD}_{G}\left(A^{\infty}\right) .
$$

Let $L, K \in \mathrm{SD}_{G}\left(T^{\infty}\right)$. A basic result from set theory yields $\sigma^{-1}(L \cup K)=\sigma^{-1}(L) \cup$ $\sigma^{-1}(K)$. Let $\sigma(v)=w_{1} w_{2}$ for some $v \in\left(B^{*} c\right)^{*}$. Since $B^{*} c$ is a prefix code, there exists a unique factorization $v=v_{1} v_{2}$ with $v_{1}, v_{2} \in\left(B^{*} c\right)^{*}$ such that $\sigma\left(v_{1}\right)=w_{1}$ and $\sigma\left(v_{2}\right)=w_{2}$. 
Thus, we conclude $\sigma^{-1}(K \cdot L)=\sigma^{-1}(K) \cdot \sigma^{-1}(L)$. Let now $K \in \mathrm{SD}_{G}\left(T^{\infty}\right)$ be a prefix code of synchronization delay $d$. We first show that $\sigma^{-1}(K)$ is a prefix code of bounded synchronization delay. Let $u, u v \in \sigma^{-1}(K)$, then $\sigma(u), \sigma(u v)=\sigma(u) \sigma(v) \in K$ and therefore $\sigma(v)=1$. This implies $v=1$ and $\sigma^{-1}(K)$ is a prefix code. We prove that $\sigma^{-1}(K)$ has synchronization delay $d+1$. The incrementation of the synchronization delay by one comes from the fact that $B^{*} c$ is not a suffix code, and thus we need another word in $B^{*} c$ to pose as a left marker. Consider $u v w \in \sigma^{-1}(K)^{*}$ with $v \in \sigma^{-1}(K)^{d+1}$ and factorize $v=v_{1} c v_{2}$ with $v_{2} \in \sigma^{-1}(K)^{d}=\sigma^{-1}\left(K^{d}\right)$. Then $\sigma(u v w)=\sigma\left(u v_{1} c\right) \sigma\left(v_{2}\right) \sigma(w)$, and by $\sigma\left(v_{2}\right) \in K^{d}$ this implies $\sigma(u v)=\sigma\left(u v_{1} c\right) \sigma\left(v_{2}\right) \in K^{*}$. Thus, $u v \in \sigma^{-1}(K)^{*}$. Let $\gamma: K^{*} \rightarrow G$ be some homomorphism and $K_{g}=K \cap \gamma^{-1}(g) \in \operatorname{SD}_{G}\left(T^{\infty}\right)$ for all $g \in G$. Inductively, $\sigma^{-1}\left(K_{g}\right) \in \mathrm{SD}_{G}\left(A^{\infty}\right)$ and $\sigma^{-1}(K)=\bigcup \sigma^{-1}\left(K_{g}\right)$. Let $\gamma^{\prime}: \sigma^{-1}(K)^{*} \rightarrow G$ be induced by $\gamma^{\prime}(u)=\gamma(\sigma(u))$. By definition of $\operatorname{SD}_{G}\left(A^{\infty}\right)$ we obtain $\gamma^{\prime-1}(1) \in \operatorname{SD}_{G}\left(A^{\infty}\right)$. However, $u_{1} \cdots u_{n} \in \sigma^{-1}\left(\gamma^{-1}(1)\right)$ if and only if $\gamma\left(\sigma\left(u_{1} \cdots u_{n}\right)\right)=1$. Furthermore, note that $\gamma\left(\sigma\left(u_{1} \cdots u_{n}\right)\right)=\gamma\left(\sigma\left(u_{1}\right)\right) \cdots \gamma\left(\sigma\left(u_{n}\right)\right)=\gamma^{\prime}\left(u_{1}\right) \cdots \gamma^{\prime}\left(u_{n}\right)=\gamma^{\prime}\left(u_{1} \cdots u_{n}\right)$. Thus, we obtain $\sigma^{-1}\left(\gamma^{-1}(1)\right)=\gamma^{\prime-1}(1) \in \operatorname{SD}_{G}\left(A^{\infty}\right)$ and $\sigma^{-1}\left(\gamma^{-1}(1)^{\omega}\right)=\gamma^{\prime-1}(1)^{\omega} \in \operatorname{SD}_{G}\left(A^{\infty}\right)$. This concludes the proof of the claim.

At this point we showed the proposition for languages $L \subseteq A^{*}$.

The last case of the proof is that $w$ contains infinitely many $c$ 's, that is, $w=c v$ with $v \in\left(B^{*} c\right)^{\omega}$. By induction, we know that $\sigma(v) \in L_{T} \cdot \gamma_{T}^{-1}(1)^{\omega} \subseteq \llbracket \sigma(v) \rrbracket_{\psi}$ for some $L_{T} \in \mathrm{SD}_{G}\left(T^{*}\right)$ and $\gamma_{T}: K_{T}^{*} \rightarrow G$ for some prefix code $K_{T} \in \mathrm{SD}_{G}\left(T^{*}\right)$ of bounded synchronization delay with $\gamma_{T}^{-1}(g) \cap K_{T} \in \mathrm{SD}_{G}\left(T^{*}\right)$. By the calculation above, there exists a $\gamma: K^{*} \rightarrow G$ with the usual properties such that $\gamma^{-1}(1)=\sigma^{-1}\left(\gamma_{T}^{-1}(1)\right)$. Let $L=\sigma^{-1}\left(L_{T}\right)$ and set $L(w)=c L \gamma^{-1}(1)^{\omega}$. It remains to show that $c L \gamma^{-1}(1)^{\omega} \subseteq \llbracket w \rrbracket_{\varphi}$. Let $c u \in c L \gamma^{-1}(1)^{\omega}$, then $\sigma(u) \in \llbracket \sigma(v) \rrbracket_{\psi}$, that is $\sigma(u) \approx_{\psi} \sigma(v)$. Since $\approx_{\psi}$ is the transitive closure of $\sim_{\psi}$, we show that $\sigma(u) \sim_{\psi} \sigma(v)$ implies $c u \approx_{\varphi} c v$ for all $u, v \in\left(B^{*} c\right)^{\omega}$ which concludes the proof. Now, let $\sigma(u)=\sigma\left(u_{1} c\right) \sigma\left(u_{2} c\right) \cdots$ and $\sigma(v)=\sigma\left(v_{1} c\right) \sigma\left(v_{2} c\right) \cdots$ such that $\psi\left(\sigma\left(u_{i} c\right)\right)=\psi\left(\sigma\left(v_{i} c\right)\right)$. As observed above, this implies $\varphi\left(c u_{i} c\right)=\varphi\left(c v_{i} c\right)$. Thus,

$$
\begin{aligned}
c u & =\left(c u_{1} c\right) u_{2}\left(c u_{3} c\right) u_{4}\left(c \cdots \sim_{\varphi}\left(c v_{1} c\right) u_{2}\left(c v_{3} c\right) u_{4}(c \cdots\right. \\
& =c v_{1}\left(c u_{2} c\right) v_{3}\left(c u_{4} c\right) \cdots \sim_{\varphi} c v_{1}\left(c v_{2} c\right) v_{3}\left(c v_{4} c\right) \cdots \\
& =c v .
\end{aligned}
$$

This implies the existence of finitely many sets $L(w) \in \mathrm{SD}_{G}\left(A^{\infty}\right)$ with $w \in L(w) \subseteq \llbracket w \rrbracket_{\varphi}$ in the case of infinitely many $c$ 's.

\section{$5 \quad$ Rees extension monoids}

We need the fact that every group contained in $\operatorname{Rees}(\mathrm{N}, \mathrm{M}, \rho)$ is contained in $N$ or in $M$.

- Lemma $12([1])$. Let $G$ be a subgroup of $\operatorname{Rees}(\mathrm{N}, \mathrm{M}, \rho)$, then there exists an embedding of $G$ into $N$ or into $M$.

Thus, Lemma 12 implies LocRees $(\mathbf{H}) \subseteq \operatorname{Rees}(\mathbf{H}) \subseteq \operatorname{Rees}(\overline{\mathbf{H}}) \subseteq \overline{\mathbf{H}}$ for any group variety $\mathbf{H}$. We want to prove equality, that is, every monoid which contains only groups in $\mathbf{H}$ is a divisor of an iterated Rees extension of groups in $\mathbf{H}$. However, we are able to prove a stronger statement using only local Rees extensions.

- Lemma 13. Let $M$ be a monoid, $N$ be a submonoid of $M$ and $c \in M$. If $N$ and $c$ generate $M$, then $M$ is a homomorphic image of the local Rees extension LocRees $\left(\mathrm{N}, \mathrm{M}_{\mathrm{c}}\right)$. 
Proof. Let $\varphi$ : LocRees $\left(\mathrm{N}, \mathrm{M}_{\mathrm{c}}\right) \rightarrow M$ be the mapping given by $\varphi(n)=n$ for $n \in N$ and $\varphi(u, x, v)=u x v$ for $(u, x, v) \in N \times M_{c} \times N$. Since

$$
\begin{aligned}
\varphi((u, x, v)(s, y, t)) & =\varphi(u, x \circ \operatorname{cvsc} \circ y, t)=\varphi(u, x v s y, t) \\
& =(u x v)(s y t)=\varphi(u, x, v) \varphi(s, y, t),
\end{aligned}
$$

$\varphi$ is a homomorphism. Obviously, $M=N \cup N M_{c} N$ and thus $\varphi$ is surjective.

A Rees decomposition of a monoid $M$ is a sequence of monoids $M_{1}, \ldots, M_{k}=M$ such that for each $1 \leq j \leq k$ we have for $M_{j}$ one of the following:

- $M_{j}$ is a group which is a divisor of $M$.

- $M_{j}$ is a divisor of a local Rees extension of a submonoid $M_{i}$ of $M_{j}$ and a local divisor $M_{\ell}$ of $M_{j}$ with $i, \ell<j$.

- Proposition 14. A finite monoid $M$ has a Rees decomposition of length at most $2^{|M|}-1$.

Proof. We prove the statement with induction on $|M|$. If $M$ is a group, we set $M_{1}=M$. This includes the base case $|M|=1$. If $M$ is not a group, we may choose a minimal generating set of $M$. Let $c$ be a nonunit of this generating set, then there exists a proper submonoid $N$ of $M$ such that $N$ and $c$ generate $M$. Since $c$ is not a unit, the local divisor $M_{c}$ is smaller than $M$, that is, $\left|M_{c}\right|<|M|$. By induction, there exist Rees decompositions $M_{1}^{\prime}, \ldots, M_{k^{\prime}}^{\prime}=N$ and $M_{1}^{\prime \prime}, \ldots, M_{k^{\prime \prime}}^{\prime \prime}=M_{c}$ with $k^{\prime}, k^{\prime \prime} \leq 2^{|M|-1}-1$. Note that every group, which is a divisor of $N$ or $M_{c}$ also is a divisor of $M$. Furthermore, $M$ is a divisor of the local Rees extension of $M_{k^{\prime}}=N$ and $M_{k^{\prime}+k^{\prime \prime}}=M_{c}$ by Lemma 13 . Therefore, choosing

- $M_{i}=M_{i}^{\prime}$ for $1 \leq i \leq k^{\prime}$

- $M_{i+k^{\prime}}=M_{i}^{\prime \prime}$ for $1 \leq i \leq k^{\prime \prime}$

- $M_{k^{\prime}+k^{\prime \prime}+1}=M$

leads to such a sequence for $M$. Since $k^{\prime}+k^{\prime \prime}+1 \leq 2 \cdot\left(2^{|M|-1}-1\right)+1=2^{|M|}-1$, the bound on $k$ holds.

The inclusion $\overline{\mathbf{H}} \subseteq \operatorname{LocRees}(\mathbf{H})$ is immediate from Proposition 14. This yields

- Theorem 15. Let $\mathbf{H}$ be a variety of finite groups. Then $\overline{\mathbf{H}}=\operatorname{LocRees}(\mathbf{H})=\operatorname{Rees}(\mathbf{H})$.

In particular, every monoid in $\overline{\mathbf{H}}$ is a divisor of an iterated Rees extension of groups in $\mathbf{H}$ by Lemma 1. We can draw the decomposition as a tree based on the decomposition of $M$ in submonoids and local divisors. We do not describe this formally but content ourselves to give an example.

- Example 16. Let $M$ be the monoid generated by $\{a, b, \delta, \sigma\}$ with the relations $a^{2}=b^{2}=$ $a b=b a=0, a \delta=a, \delta \sigma=\sigma \delta^{2}, \delta^{3}=1, \sigma^{2}=1$ and $d \delta=\delta d, d \sigma=\sigma d$ with $d \in\{a, b\}$. The subgroup generated by $\delta$ and $\sigma$ is the symmetric group $\mathfrak{S}_{3}$; it is solvable but not abelian. The monoid $M$ is syntactic for the language $L$ which is a union of $L_{a}$ and $L_{b}$. The language $L_{a}$ is the set of all words $u a v$ with $u v \in\{\delta, \sigma\}^{*}$ and the sign of the permutation $u v$ evaluates to -1 . The language $L_{b}$ is the set of all words $u b v$ with $u v \in\{\delta, \sigma\}^{*}$ and $u v$ evaluates in $\mathfrak{S}_{3}$ to $\delta$. The decomposition in local Rees extensions from Proposition 14 is depicted in Figure 1. Here $M[a, \sigma, \delta]$ denotes the submonoid generated by $\{a, \sigma, \delta\}$. In particular, this yields

$$
M \preceq \operatorname{Rees}\left(\operatorname{Rees}\left(\mathfrak{S}_{3}, \operatorname{Rees}\left(\mathbb{Z} / 2 \mathbb{Z},\{1\}, \rho_{1}\right), \rho_{2}\right), \operatorname{Rees}\left(\mathfrak{S}_{3},\{1\}, \rho_{3}\right), \rho_{4}\right)
$$

for some $\rho_{1}, \rho_{2}, \rho_{3}, \rho_{4}$ by Lemma 1 . 


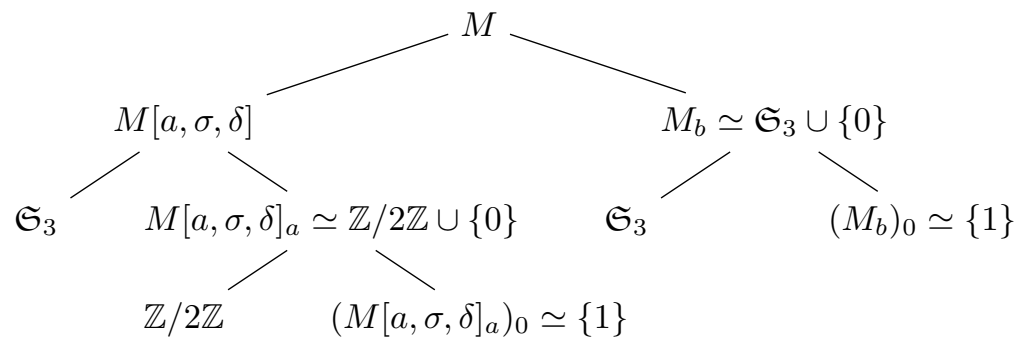

Figure 1 Decomposition tree of the monoid in Example 16.

\section{$6 \quad$ Applications}

An application of Proposition 14 is the solution to an open problem of Almeida and Klíma. Let $\mathbf{U}$ and $\mathbf{V}$ be varieties. Let $\operatorname{Rees}(\mathbf{U}, \mathbf{V})$ be the variety generated by $\operatorname{Rees}(\mathrm{N}, \mathrm{M}, \rho)$ for $N \in \mathbf{U}$ and $M \in \mathbf{V}$. Note that in general $\operatorname{Rees}(\mathbf{V}) \neq \operatorname{Rees}(\mathbf{V}, \mathbf{V})$. However $\operatorname{Rees}(\mathbf{V})$ can be defined as the limit of this operation. Let $\mathbf{V}_{i}=\operatorname{Rees}\left(\mathbf{V}_{\mathrm{i}-1}, \mathbf{V}_{\mathrm{i}-1}\right)$ and $\mathbf{V}_{0}=\mathbf{V}$, then

$$
\operatorname{Rees}(\mathbf{V})=\bigcup_{i \in \mathbb{N}} \mathbf{V}_{i}
$$

The variety $\operatorname{Rees}(\mathbf{U}, \mathbf{V})$ has recently been introduced by Almeida and Klíma under the name of bullet operation [1]. They defined a variety $\mathbf{V}$ to be bullet idempotent if $\mathbf{V}=\operatorname{Rees}(\mathbf{V}, \mathbf{V})$ and they asked whether there are varieties apart from $\overline{\mathbf{H}}$ which are bullet idempotent. Using our decomposition above, we prove that the answer to this question is "No".

- Theorem 17. Let $\mathbf{V}$ be a bullet idempotent variety and let $\mathbf{H}=\mathbf{V} \cap \mathbf{G}$, then $\mathbf{V}=\overline{\mathbf{H}}$.

Proof. Since $\overline{\mathbf{H}}$ is the maximal variety with $\overline{\mathbf{H}} \cap \mathbf{G}=\mathbf{H}$, we have $\mathbf{V} \subseteq \overline{\mathbf{H}}$. Let $M \in \overline{\mathbf{H}}$. Inductively, we may assume that every proper divisor of $M$ is in $\mathbf{V}$. If $M$ is a group, then $M \in \mathbf{H}$ and thus $M \in \mathbf{V}$. Thus, there exists an nonunit element $c \in M$ and a proper submonoid $N$ of $M$ such that $N$ and $c$ generate $M$. By Lemma 13, $M$ is a divisor of $\operatorname{LocRees}\left(\mathrm{N}, \mathrm{M}_{\mathrm{c}}\right)$, and since $N, M_{c} \in \mathbf{V}$ and $\mathbf{V}=\operatorname{Rees}(\mathbf{V}, \mathbf{V})$ we obtain $M \in \mathbf{V}$.

Let $\left(\mathrm{FO}+\mathrm{MOD}_{q}\right)[<]$ be the fragment of first-order sentences which only use first-order quantifiers, modular quantifiers of modulus $q$ and the predicate $<$. Then the following theorem holds.

- Corollary 18. $\left(\mathrm{FO}+\mathrm{MOD}_{q}\right)[<]\left(A^{\infty}\right)=\mathrm{SD}_{\mathbf{S o l}_{q}}\left(A^{\infty}\right)$

Proof. By [20], see also [19] for a complete treatise, $\left(\mathrm{FO}+\mathrm{MOD}_{q}\right)[<]$ describes the family of all regular languages such that every group in the syntactic monoid is a solvable group of cardinality dividing a power of $q$, that is the languages in $\mathbf{S o l}_{q}$. Theorem 4 then implies the stated equality.

The same language class has been described by Straubing with another operation, counting how many prefixes are in a given language, which resembles more closely the counting modulo $q[18]$.

\section{Summary}

Our main theorem Theorem 4 states $\overline{\mathbf{H}}\left(A^{\infty}\right)=\operatorname{SD}_{\mathbf{H}}\left(A^{\infty}\right)$. An overview over the contributions for $\overline{\mathbf{H}}$ is given in Table 1 . 
Table 1 Overview of existing and new language characterizations of $\overline{\mathbf{H}}$.

\begin{tabular}{|l|c|c|c|c|c|}
\hline & $\overline{\mathbf{1}}$ & $\overline{\mathbf{A b}}$ & $\overline{\text { Sol }}$ & $\overline{\mathbf{S o l}_{q}}$ & $\overline{\mathbf{H}}$ \\
\hline finite words & {$[17]$} & {$[16]$} & {$[18]$, new } & {$[18]$, new } & new, unless $\mathbf{H} \subseteq \mathbf{A b}$ \\
\hline$\omega$-words & {$[5]$} & new & new & new & new, unless $\mathbf{H}=\mathbf{1}$ \\
\hline
\end{tabular}

As a byproduct we were able to give a simple decomposition of the monoids in $\overline{\mathbf{H}}$ as local Rees extensions and groups in $\mathbf{H}$, using only exponentially many operations.

\section{References}

1 Jorge Almeida and Ondřej Klíma. On the irreducibility of pseudovarieties of semigroups. Journal of Pure and Applied Algebra, 220(4):1517-1524, 2016. doi:10.1016/j .jpaa. 2015. 09.015.

2 André Arnold. A syntactic congruence for rational $\omega$-languages. Theoretical Computer Science, 39:333-335, 1985.

3 Jean-Camille Birget and John L. Rhodes. Almost finite expansions of arbitrary semigroups. Journal of Pure and Applied Algebra, 32(3):239-287, 1984.

4 Jean-Camille Birget and John L. Rhodes. Group theory via global semigroup theory. Journal of Algebra, 120(2):284-300, 1989. doi:10.1016/0021-8693(89)90199-3.

5 Volker Diekert and Manfred Kufleitner. Omega-rational expressions with bounded synchronization delay. Theory Comput. Syst., 56:686-696, 2015.

6 Volker Diekert and Manfred Kufleitner. A survey on the local divisor technique. Theoretical Computer Science, 610:13-23, 2015. doi:10.1016/j.tcs.2015.07.008.

7 Volker Diekert, Manfred Kufleitner, and Pascal Weil. Star-free languages are Church-Rosser congruential. Theoretical Computer Science, 454:129-135, 2012. doi :10.1016/j.tcs . 2012. 01.028.

8 Volker Diekert and Grzegorz Rozenberg, editors. The Book of Traces. World Scientific, Singapore, 1995.

9 Samuel Eilenberg. Automata, Languages, and Machines, volume B. Academic Press, New York and London, 1976.

10 Dominique Perrin. Recent results on automata and infinite words. In Mathematical foundations of computer science, 1984 (Prague, 1984), volume 176 of Lecture Notes in Comput. Sci., pages 134-148. Springer, Berlin, 1984.

11 Dominique Perrin and Jean-Éric Pin. Infinite words, volume 141 of Pure and Applied Mathematics. Elsevier, Amsterdam, 2004.

12 Jean-Éric Pin. Varieties of Formal Languages. North Oxford Academic, London, 1986.

13 John Rhodes and Dennis Allen. Synthesis of the classical and modern theory of finite semigroups. Advances in Mathematics, 11(2):238-266, 1973. doi:10.1016/0001-8708(73) 90010-8.

14 John L. Rhodes and Benjamin Steinberg. The q-theory of finite semigroups. Springer Monographs in Mathematics. Springer, 2009.

15 Marcel-Paul Schützenberger. On finite monoids having only trivial subgroups. Information and Control, 8:190-194, 1965.

16 Marcel-Paul Schützenberger. Sur les monoides finis dont les groupes sont commutatifs. Rev. Française Automat. Informat. Recherche Opérationnelle Sér. Rouge, 8(R-1):55-61, 1974.

17 Marcel-Paul Schützenberger. Sur certaines opérations de fermeture dans les langages rationnels. In Symposia Mathematica, Vol. XV (Convegno di Informatica Teorica, INDAM, Roma, 1973), pages 245-253. Academic Press, 1975. 


\section{9:14 Characterizing Regular Languages Using Prefix Codes}

18 Howard Straubing. Families of recognizable sets corresponding to certain varieties of finite monoids. Journal of Pure and Applied Algebra, 15(3):305-318, 1979.

19 Howard Straubing. Finite Automata, Formal Logic, and Circuit Complexity. Birkhäuser, Boston, Basel and Berlin, 1994.

20 Howard Straubing, Denis Thérien, and Wolfgang Thomas. Regular languages defined with generalized quantifiers. Inform. and Comput., 118(2):289-301, 1995.

21 Wolfgang Thomas. Automata on infinite objects. In J. van Leeuwen, editor, Handbook of Theoretical Computer Science, chapter 4, pages 133-191. Elsevier Science Publishers B. V., 1990.

22 Thomas Wilke. An algebraic theory for regular languages of finite and infinite words. International Journal of Algebra and Computation, 3(4):447-489, 1993. 